\title{
Caught Between External Pressures and Internal Battles: Psychosocial Factors Affecting Gestational Weight Gain - A Scoping Review
}

\author{
Unsa Athar ${ }^{1}$, Noor Ul Ain Daud ${ }^{1}$, Warda A. Khan ${ }^{1}$, Amna Khalid ${ }^{1}$, Seemab Imtiaz Gill ${ }^{2}$ \\ 1. Community Health Sciences, Shalamar Medical and Dental College, Lahore, PAK 2. Internal Medicine, King Edward \\ Medical University, Lahore, PAK
}

Corresponding author: Unsa Athar, unsa.athar10@gmail.com

\begin{abstract}
Many physical factors (including maternal comorbidities) affecting gestational weight gain (GWG) have been widely studied; however, the psychosocial aspects pertaining to this need to be put under the microscope, especially in countries characterized by low indicators of socioeconomic development. Gaining and maintaining an adequate amount of weight during pregnancy is important to prevent premature deliveries, fetal demise, fetal macrosomia, shoulder dystocia during delivery, emergency cesarean sections, postpartum weight retention, childhood obesity, etc. A scoping review of the articles published in the last five years has revealed that perinatal outcomes like gestational weight are influenced by certain psychosocial factors, including, but not limited to, intimate partner violence, lack of social support and recognition, financial distress, household food insecurity, chronic stress and depression related to pregnancy, eating pathologies, and low self-esteem. Employing a multi-disciplinary approach, which involves seeking the help of psychiatrists/psychologists, obstetricians, nutritionists, and public health specialists, can help us mitigate undesirable outcomes related to inadequate and excessive weight gain during pregnancy. More intervention-based research focusing on psychosocial factors relating to GWG is needed in regions like South Asia, which is associated with low indicators of socioeconomic development.
\end{abstract}

Review began 02/14/2021 Review ended 02/15/2021 Published 02/22/2021

๑) Copyright 2021 Athar et al. This is an open access article distributed under the terms of the Creative Commons Attribution License CC-BY 4.0., which permits unrestricted use, distribution, and reproduction in any medium, provided the original author and source are credited.
Categories: Obstetrics/Gynecology, Psychiatry, Public Health

Keywords: low socioeconomic, obesity, pre-pregnancy body mass index, gestational weight gain, cesarean section, macrosomia, perinatal outcomes, psychosocial factors, rate of gestational weight gain, weight gain in pregnancy, inadequate weight gain in pregnancy

\section{Introduction And Background}

Many factors contribute toward helping women maintain a healthy physical and mental state during pregnancy and successfully carry it to term, preferably without any complications for the mother and fetus alike. Factors affecting the physical health of the mother, and hence the fetus that is carried, such as the presence of underlying comorbidities like obesity, diabetes, hypertension, dyslipidemias, metabolic syndrome, or other physical diseases like systemic inflammation, vascular dysfunction, which might affect the mother in the pre-gestational and gestational periods, have been extensively studied [1]. However, the psychosocial factors responsible for maternal health and their potential impact on the well-being of both the mother and fetus remain largely understudied; hence, a clear association between them has not been established [2].

Regarding the evolution of medical perceptions toward gestational weight gain (GWG), clinicians used to believe that a weight gain of more than 9 kilograms can lead to detrimental effects [3]. In the early 1930s, more research was conducted on the subject, and by the 1970s, it was established that an adequate weight gain maintained by a healthy diet and activity is essential for favorable outcomes for the mother and the fetus [3]. The Institute of Medicine (IOM) and National Research Council (NRC) recommend an ideal GWG of 11.5-16 kilograms for women who have a normal pre-pregnancy weight/body mass index [4]. Gaining and maintaining an adequate amount of weight in pregnancy is important because the lack of it leads to premature deliveries owing to small-for-gestational-age weight in the babies, and even fetal demise. On the other hand, excessive weight gain also has numerous maternal and fetal repercussions, such as fetal macrosomia, shoulder dystocia during delivery, emergency cesarean sections, postpartum weight retention, and childhood obesity [5].

Even though many physical factors (including maternal comorbidities) affecting GWG have been studied, the psychosocial aspects of it need to be delved into in a deeper manner, especially in countries with low socioeconomic indices. A study performed in Pakistan [6] showed that depression experienced by mothers during pregnancy is chronic in nature and known to persist for as many as three to four years following childbirth. This period is considered crucial for the emotional and cognitive development of an infant, which can be adversely affected by the lack of mental well-being in mothers. Maternal depression can also lead to poor physical outcomes in children, such as stunting and underweight, emphasizing the influence of maternal mental health on the physical and psychological aspects of a child's health [6].

With this context in mind, our objective in conducting this review was to scope through the existing 
evidence about psychosocial factors affecting maternal health, in order to emphasize the need for and understand the extent of further research that needs to be performed on this topic.

\section{Review \\ Methodology}

An evidence-based methodological protocol [7] was employed to look for peer-reviewed articles. The authors were divided into two teams: A and B. Team A and Team B performed the first stages of the scoping separately. The following databases were used depending upon the ease of access: PubMed, PubMed Central, ProQuest, PsychINFO, Online Wiley Library, and Ovid. The Medical Subject Headings (MeSH) terms employed to conduct the search were as follows: 'psychosocial factors AND gestational weight gain,' 'inadequate gestational weight gain,' 'excessive gestational weight,' and 'rate of weight gain in pregnancy.' A total of 1,532 results were generated. A filter was then applied to select only those articles that were published from 2015 to 2020 . The studies involving animals and those published in any language other than English along with repeated articles were eliminated. Articles that dealt with physical comorbidities concerning GWG, such as diabetes mellitus, as well as those involving women with a previous history of mental or psychiatric diseases, were excluded after reading through their methodologies (full-text screening). Studies involving previously diagnosed psychiatric or physical comorbidities were excluded so that their confounding effects on GWG could be removed. The population in the included studies had a singleton pregnancy, without any fetal anomalies. The two teams then matched and compared their results. Duplicates were removed and disagreements were resolved through discussions and by arriving at mutual agreements. All these factors narrowed down the number of concerned articles to a total of nine, the quality of which were assessed by using the Strengthening the Reporting of Observational Studies in Epidemiology (STROBE) [8] guidelines. This methodology adopted is illustrated in Figure 1. The articles that were ultimately included for analysis $(n=9)$ were thoroughly studied by the authors. The focus was placed more on the methodology than the results as this was a scoping review. The different types of studies that were included are shown in Figure 2. 


\section{Cureus}



FIGURE 1: Selection of articles 


\title{
Cureus
}

\section{Types of studies $(n=9)$}



\section{Cross sectional studies}

\author{
Review articles
}

Cohort study

Secondary data derived from a cohort study

Secondary data derived from RCT

FIGURE 2: Type/methodology of selected articles $(n=9)$

RCT: randomized controlled trial

\section{Results and discussion}

The primary outcomes (as depicted in the titles) that were studied, the bio-statistical tests applied, the population included in the articles [9-15], and their limitations are summarized in Table 1. There was a consensus among all the articles that there is a dearth of research on the impact of psychosocial factors and their relation with gestational weight. The IOM and NRC in 2009 declared that there is a dire need for research that can determine the interventions aimed at psychosocial elements leading to excessive or inadequate weight gain [4]. Almost a decade later, we are still facing a situation where there is a significant lack of work done on the aspect of psychosociology, as there still remains a stigma concerning mental health associated with cultural norms. To get an idea of where we stand today, this scoping review focused on the research methodology practiced in the last five years, in order to lay the groundwork for further intervention-based studies. The picture that has emerged after analyzing the selected articles clearly exhibited women caught between a society that is unwilling to provide support and the distortions of their own cognition by which they view themselves. Psychosocial factors that determine the inadequacy and/or excess of GWG can be classified into various types, as shown in Figure 3. 


\section{Cureus}

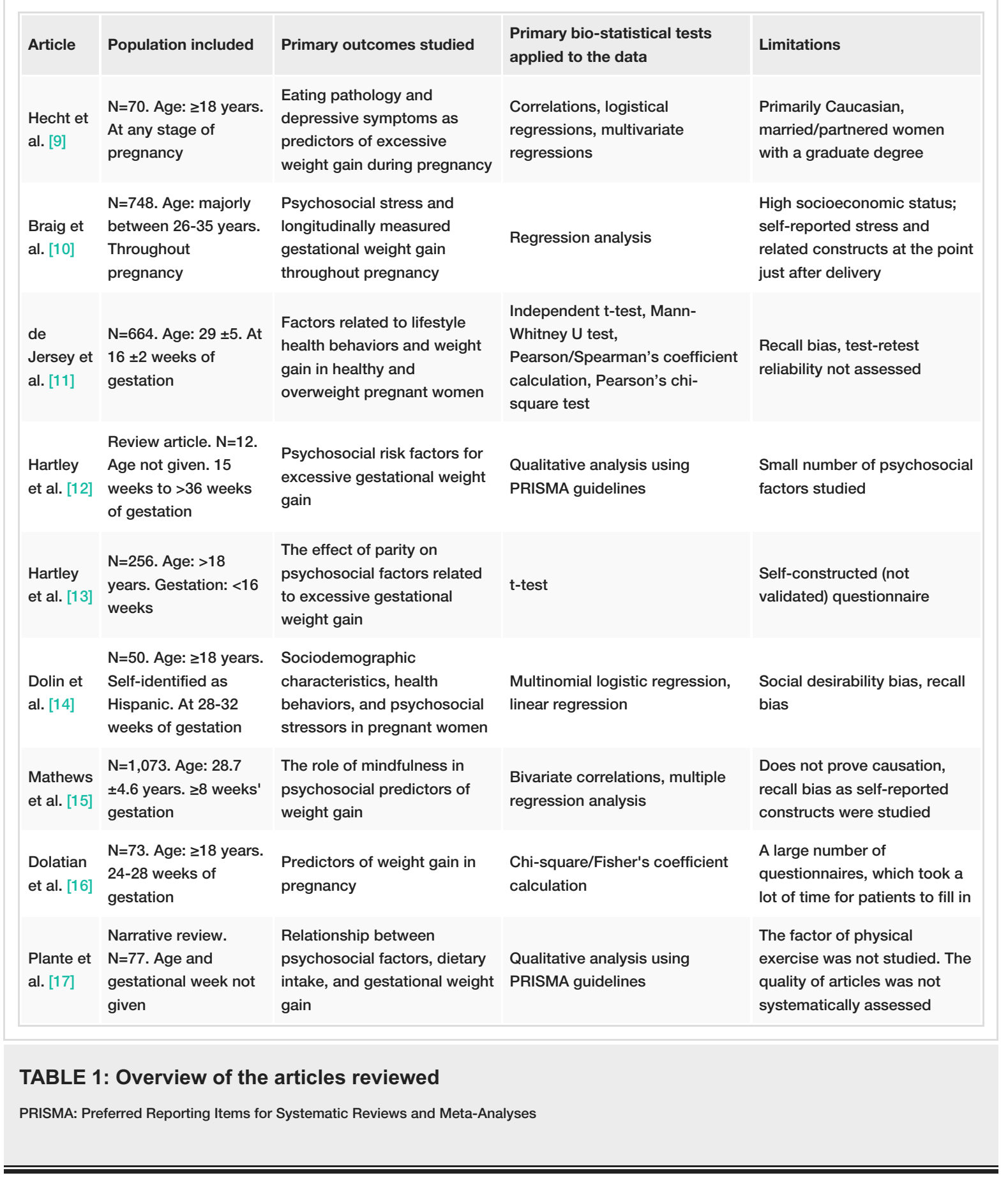






FIGURE 3: Psychosocial factors related to the inadequacy and/or excess of gestational weight gain

\section{Social elements}

Intimate Partner Violence and Lack of Social Support From Loved Ones

An analysis of variables related to pre-pregnancy BMI revealed that women who reported a lack of social support showed signs of improper GWG [13,14]. During their pregnancy, women felt isolated from friends and family, describing themselves as 'not being appreciated adequately in social circles.' They also experienced a stressful environment created by their neighbors' constant interference in their lives [14]. In addition to that, violence committed by intimate partners also bred an incompatible environment for a healthy pregnancy, which consequently impacted the rate of weight gain in pregnancy [15]. Dolatian et al. [16] created a conceptual framework showing the role of violence, giving it a central role in the rate of weight gain in pregnancy. Violence at the hands of intimate partners and other family members, particularly during pregnancy, has been detrimental to women's well-being since the advent of time [17]. Violence can be in different forms, including physical, sexual, and emotional. A study in Malaysia has reported that 
women who encounter violence during pregnancy show a higher odds ratio for developing anemia, inadequate weight gain, urinary tract infections, premature rupture of membranes, and antepartum hemorrhage [18].

Financial Distress and Household Food Insecurity

Through various analytical methods, it was observed that financial distress leads to statistically significant effects $(\mathrm{p}<0.05)$ on the rate of GWG [10,14]. Inadequate consumption of healthy food due to poor socioeconomic status (food insecurity) and the added burden of stress related to unemployment (inability to pay rent/bills) acted as a barrier for a healthy GWG [14]. Such perceived distress due to financial burden was also found to be related to both excessive $(\mathrm{p}=0.02)$ and inadequate weight gain in a study performed as a secondary data analysis of the Gestational Diabetes's Effects on Moms trial conducted at Kaiser Permanente - Northern California (KPNC).

\section{Psychiatric/psychological elements}

Despite having no previously diagnosed psychiatric illness, many women experience depression, anxiety, and stress during pregnancy, which impacts their weight gain $[9-13,15,16]$.

Chronic Stress and Depression

Stress has been long known as one of the many culprits behind excessive weight gain. Stress, particularly during pregnancy, disrupts the functioning of the hypothalamus-pituitary axis (HPA). This in turn increases the level of cortisol in the body, which redistributes fatty acid deposition to the center, and also increases 'cravings' that lead to over-eating [19,20]. Depressive symptoms and chronic stress have been unanimously found to be a predictor of excessive and/or inadequate weight gain [9-16]. Dolin et al. have reported that $11 \%$ of pregnant Hispanic immigrants showed perinatal depression and excessive weight gain, as assessed by a validated Patient-Health Questionnaire-9 tool [14]. The relationship between depression and GWG is, however, complicated and needs to be analyzed and studied more so that timely interventions and screening measures can be implemented. It is noteworthy that the practice of mindfulness techniques has shown an inverse relationship with the development of excessive weight gain [15]. The practice of mindfulness techniques by pregnant women has been observed to help overcome many hurdles like physical discomfort and psychological distress [21]. Getting a handle on mindfulness practices can help pregnant women become more self-aware of their condition, which in turn can help them keep their weight in check based on IOM guidelines.

\section{Eating Disorders}

Eating pathologies are also associated with excessive GWG worldwide [9]. Many pregnant women go through a phase of 'emotional eating' and cravings, mostly because of increased cortisol levels [10], which can lead to weight gain in excess of what is recommended by IOM. Dolin et al. [14] have reported a lower risk of excessive GWG in women who ate breakfast daily during their pregnancy. More than three hours of screen time and skipping breakfast turned out to be predictive of improper weight gain. Food insecurity is defined as the inability to access nutritious food due to poor living conditions and low economic status. In diverse urban populations, every one in 10 women is affected by food insecurity during their pregnancy [22]. The condition is even worse among rural populations and those living in urban slums. This food insecurity contributes to fostering eating pathologies [16]. Other factors that were found to cause eating disorders included low social support and unhappy marital relationships [17]. Following a healthy, balanced diet plan leads to a positive influence on the overall quality of life during pregnancy and thus ensures adequate weight gain [23]. To ensure that pregnant women get the full benefit of a healthy diet, psychosocial factors that contribute to eating disorders should be managed in a timely manner.

\section{Low Self-Esteem}

Women exhibiting sufficient self-efficacy were observed to experience adequate weight gain. Having a positive attitude towards weight management also showed an association with proper GWG [11]. Pregnant women who displayed low self-esteem and greater dissatisfaction with their bodies, characterizing themselves as 'fat' or 'unattractive', showed a higher risk of inadequate weight gain leading to undesirable perinatal outcomes [17]. The above observations clearly indicate the role psychosocial factors play in mediating an adequate GWG. Interventional approaches by obstetricians and physicians targeted to tailor to these psychosocial factors can improve maternal and neonatal outcomes. There is an apparent dearth of such research in South Asian countries, where psychosocial factors are much more prevalent compared to first-world countries. Social ills like child marriage have been shown to drastically affect adequate GWG. The prevalence of child marriage is high in Southeast Asia, the highest incidence being recorded in Bangladesh where approximately $52 \%$ of the girls are married before the age of 18 and $18 \%$ are married before 15 years of age. India is at a close second (47\%), followed by Nepal (37\%), Afghanistan (33\%), and Pakistan (21\%) [24]. A study on child marriages in Bangladesh has proposed that public health interventions aimed towards ending child marriages can bring about good perinatal outcomes, as child marriages were associated with inadequate gestational weight in the second and third trimesters [25]. 


\section{Recommendations}

Public health specialists need to be consulted in order to implement strategies at government levels to target the social elements discussed in this article. The availability of nutritious food and access to healthcare services need to be ensured. The spirit of social support must be fostered by providing the services of family counseling. Implementing strict laws by law enforcement agencies can be helpful in preventing child marriages and intimate partner violence. The plan of action for these recommendations is presented in Table 2 .

\section{Recommendation}

Easy access to holistic healthcare services for pregnant patients

Easy access to nutritious food

Availability of counseling

Public health interventions

Availability of safe spaces

\section{Plan of action}

Training more birth-attendants and lady health workers/visitors in the fields of mental health and nutrition

Availability of ration cards for pregnant females to buy fortified foods from utility stores

Free-of-cost appointments with psychiatrists and psychologists to help deal with stress/anxiety/depression/eating disorders related to or occurring in pregnancy in a timely manner

More quantitative research (like cohort studies and randomized controlled trials) is needed after the implementation of the above

Functional shelters must be available for pregnant females experiencing abuse from an intimate partner

TABLE 2: Recommendations and plan of action

\section{Conclusions}

A narrative review of the articles published in the last five years (2015-2020) has revealed the scope of research on GWG. Inadequate and excessive weight gain during pregnancy can have detrimental effects on both the mother and the child. Based on our findings, GWG is influenced by certain psychosocial factors, including, but not limited to, intimate partner violence, lack of social support and recognition, financial distress, household food insecurity, chronic stress and depression related to pregnancy, eating pathologies, and low self-esteem. Employing a multi-disciplinary approach, which involves using the services of psychiatrists/psychologists, obstetricians, trained birth attendants, nutritionists, and public health specialists, can help us mitigate the undesirable outcomes of inadequate and excessive weight gain. More intervention-based research targeting psychosocial factors is needed in regions characterized by low socioeconomic indicators, such as South Asia.

\section{Additional Information \\ Disclosures}

Conflicts of interest: In compliance with the ICMJE uniform disclosure form, all authors declare the following: Payment/services info: All authors have declared that no financial support was received from any organization for the submitted work. Financial relationships: All authors have declared that they have no financial relationships at present or within the previous three years with any organizations that might have an interest in the submitted work. Other relationships: All authors have declared that there are no other relationships or activities that could appear to have influenced the submitted work.

\section{Acknowledgements}

All authors have contributed equally to the formulation of this article. We would, however, like to place on record our special thanks to Professor Dr. Tahseen Haider Kazmi (Professor of Community Health Sciences) for letting his students think outside the box.

\section{References}

1. Black RE, Victora CG, Walker SP, et al.: Maternal and child undernutrition and overweight in low-income and middle-income countries. Lancet. 2013, 382:427-451. 10.1016/S0140-6736(13)60937-X

2. Gentile S: Untreated depression during pregnancy: short- and long-term effects in offspring. A systematic review. Neuroscience. 2017, 342:154-166. 10.1016/j.neuroscience.2015.09.001

3. Shenassa ED, Kinsey C, Moser Jones M, Fahey J: Gestational weight gain: historical evolution of a contested health outcome. Obstet Gynecol Surv. 2017, 72:445-453. 10.1097/OGX.0000000000000459

4. Institute of Medicine (US) and National Research Council (US) Committee to Reexamine IOM Pregnancy Weight Guidelines: Weight Gain During Pregnancy: Reexamining the Guidelines. National Academies Press, Washington, DC; 2009. 10.17226/12584

5. Viswanathan M, Siega-Riz AM, Moos MK, et al: Outcomes of maternal weight gain, evidence report/technology assessment, No. 168. (2008). Accessed: February 21, 2021: 
https://www.researchgate.net/profile/Andrea_Deierlein2/publication/5231039_Outcomes_of_maternal_weight_gain/links/00

6. Husain N, Zulqernain F, Carter LA, et al.: Treatment of maternal depression in urban slums of Karachi, Pakistan: a randomized controlled trial (RCT) of an integrated maternal psychological and early child development intervention. Asian J Psychiatr. 2017, 29:63-70. 10.1016/j.ajp.2017.03.010

7. Arksey H, O’Malley L: Scoping studies: towards a methodological framework . Int J Soc Res Methodol. 2007, 8:19-32. 10.1080/1364557032000119616

8. von Elm E, Altman DG, Egger M, Pocock SJ, Gøtzsche PC, Vandenbroucke JP; STROBE Initiative: The strengthening the reporting of observational studies in epidemiology (STROBE) statement: guidelines for reporting observational studies. J Clin Epidemiol. 2008, 61:344-349. 10.1016/j.jclinepi.2007.11.008

9. Hecht LM, Schwartz N, Miller-Matero LR, Braciszewski JM, Haedt-Matt A: Eating pathology and depressive symptoms as predictors of excessive weight gain during pregnancy. J Health Psychol. 2020, 17:1359105320913934. 10.1177/1359105320913934

10. Braig S, Logan CA, Reister F, Rothenbacher D, Genuneit J: Psychosocial stress and longitudinally measured gestational weight gain throughout pregnancy: the Ulm SPATZ Health Study. Sci Rep. 2020, 10:1996. 10.1038/s41598-020-58808-8

11. de Jersey SJ, Mallan K, Callaway L, Daniels LA, Nicholson JM: A cross sectional comparison of predisposing reinforcing and enabling factors for lifestyle health behaviours and weight gain in healthy and overweight pregnant women. Matern Child Health J. 2017, 21:626-635. 10.1007/s10995-016-2148-0

12. Hartley E, McPhie S, Skouteris H, Fuller-Tyszkiewicz M, Hill B: Psychosocial risk factors for excessive gestational weight gain: a systematic review. Women Birth. 2015, 28:e99-e109. 10.1016/j.wombi.2015.04.004

13. Hartley E, McPhie S, Fuller-Tyszkiewicz M, Hill B, Skouteris H: Psychosocial factors and excessive gestational weight gain: the effect of parity in an Australian cohort. Midwifery. 2016, 32:30-37. 10.1016/j.midw.2015.09.009

14. Dolin CD, Gross RS, Deierlein AL, et al.: Predictors of gestational weight gain in a low-income Hispanic population: sociodemographic characteristics, health behaviors, and psychosocial stressors. Int J Environ Res Public Health. 2020, 17:352. 10.3390/ijerph17010352

15. Matthews J, Huberty J, Leiferman J, Buman M: Psychosocial predictors of gestational weight gain and the role of mindfulness. Midwifery. 2018, 56:86-93. 10.1016/j.midw.2017.10.008

16. Dolatian M, Sharifi N, Mahmoodi Z, Fathnezhad-Kazemi A: Weight gain during pregnancy and its associated factors: a path analysis. Nurs Open. 2020, 17:1568-1577. 10.1002/nop2.539

17. Plante AS, Lemieux S, Labrecque M, Morisset AS: Relationship between psychosocial factors, dietary intake and gestational weight gain: a narrative review. J Obstet Gynaecol Can. 2019, 41:495-504. 10.1016/j.jogc.2018.02.023

18. Khaironisak H, Zaridah S, Hasanain FG, Zaleha MI: Prevalence, risk factors, and complications of violence against pregnant women in a hospital in Peninsular Malaysia. Women Health. 2017, 57:919-941. 10.1080/03630242.2016.1222329

19. Kubo A, Ferrara A, Brown SD, et al.: Perceived psychosocial stress and gestational weight gain among women with gestational diabetes. PLoS One. 2017, 12:e0174290. 10.1371/journal.pone.0174290

20. Adam TC, Epel ES: Stress, eating and the reward system. Physiol Behav. 2007, 91:449-458. 10.1016/j.physbeh.2007.04.011

21. Olson KL, Emery CF: Mindfulness and weight loss: a systematic review. Psychosom Med. 2015, 77:59-67. 10.1097/PSY.0000000000000127

22. Cheu LA, Yee LM, Kominiarek MA: Food insecurity during pregnancy and gestational weight gain. Am J Obstet Gynecol MFM. 2020, 2:100068. 10.1016/j.ajogmf.2019.100068

23. Bellisle F: Assessing various aspects of the motivation to eat that can affect food intake and body weight control. (Article in French). Encephale. 2009, 35:182-185. 10.1016/j.encep.2008.03.009

24. United Nations Population Fund: Marrying Too Young: End Child Marriage . (2012). Accessed: February 21, 2021: https://www.unfpa.org/sites/default/files/pub-pdf/MarryingTooYoung.pdf.

25. Rahman ML, Kile ML, Rodrigues EG, et al.: Prenatal arsenic exposure, child marriage, and pregnancy weight gain: associations with preterm birth in Bangladesh. Environ Int. 2018, 112:23-32. 10.1016/j.envint.2017.12.004 